\title{
Anti-claudin 18.2 antibody as new targeted therapy for advanced gastric cancer
}

\author{
Prabhsimranjot Singh ${ }^{1}$, Sudhamshi Toom ${ }^{2}$ and Yiwu Huang ${ }^{3^{*}}$
}

\begin{abstract}
Targeted therapy and immunotherapy have revolutionized treatment of various cancers in the past decade. Despite targeted therapy with trastuzumab in Her2-positive gastric cancer patients, survival has been dismal, mostly due to disease progression and toxicity related to the treatments. One area of active development is looking for ideal monoclonal antibodies (IMAB) specific to the proteins only on the tumor and hence avoiding unnecessary side effects. Claudin proteins with isoform 2 are one such protein, specific for several cancers, particularly gastric cancer and its metastases, leading to the development of anti-claudin 18.2 specific antibody, claudiximab. This review will highlight the latest development of claudiximab as first in class IMAB for the treatment of gastric cancer.
\end{abstract}

Keyword: Gastric cancer, Claudiximab, IMAB362, Targeted therapy, Anti-claudin antibody

\section{Background}

Gastric cancer is one of the most common cancers worldwide, the fourth (in males) and fifth (in females) most common causes of cancer-related deaths in the developed world. An estimated 951,600 new stomach cancer cases and 723,100 deaths occurred in 2012. The incidence of gastric cancer varies widely according to geographic region [1]. The majority of patients with gastric cancer are often diagnosed in the advanced stage of the disease. Early stages of gastric cancer are potentially curable with radical gastrectomy, although approximately $50 \%$ recur [2]. Adjuvant chemotherapy and chemoradiation have led to improvement in overall survival (OS) [3]. Advanced gastric cancer is not curable and treatment currently is palliative chemotherapy conferring a median survival time of 8-10 months [4]. Multiple new chemotherapy regimens are studied with improved response rates and tolerability; however, the 5-year survival rates are dismal.

Immunotherapy and targeted agents like trastuzumab, ramucirumab, and tyrosine kinase inhibitors have revolutionized the treatments of various cancer including gastric cancer [5-16]. With the advent of targeted therapy, different molecules targeting different pathways

\footnotetext{
*Correspondence: Jhuang@maimonidesmed.org

${ }^{3}$ Division of Hematology/Oncology, Maimonides Medical Center, Brooklyn, NY, USA

Full list of author information is available at the end of the article
}

were developed for the treatment of gastric cancer. In this review, we outline recently developed molecularly targeted therapy against claudin receptors-claudiximab (previously IMAB362). Claudiximab is first-in-class chimeric monoclonal antibody-IMAB (ideal monoclonal antibody), for the treatment of gastric cancer. IMABs bind to cancer-selective targets that are predominantly expressed in tumor cells and show little or no expression in healthy tissues. This unique cancer-cell selectivity of IMABs allows for maximal anticancer potency while diminishing toxicity. They have broader therapeutic window allowing optimal dosing.

\section{Claudin proteins}

Claudins are a family of proteins, first described by Shorichiro Tsukita et al. in 1998 which form the important components of the tight cell junctions [17]. They establish a paracellular barrier which controls the flow of molecules between the cells. The transmembrane domains of claudins include a $\mathrm{N}$-terminus and a C-terminus in the cytoplasm (Fig. 1). Different claudins are expressed on different tissues, their altered function has linked to formation of cancers of respective tissues $[18,19]$. Claudin-1 expression has been shown to have prognostic value in colon cancer [20], claudin-18 in gastric cancer [21], and claudin-10 in hepatocellular carcinoma [22]. Claudins, being surface proteins, represent a useful target for various therapeutic strategies. 


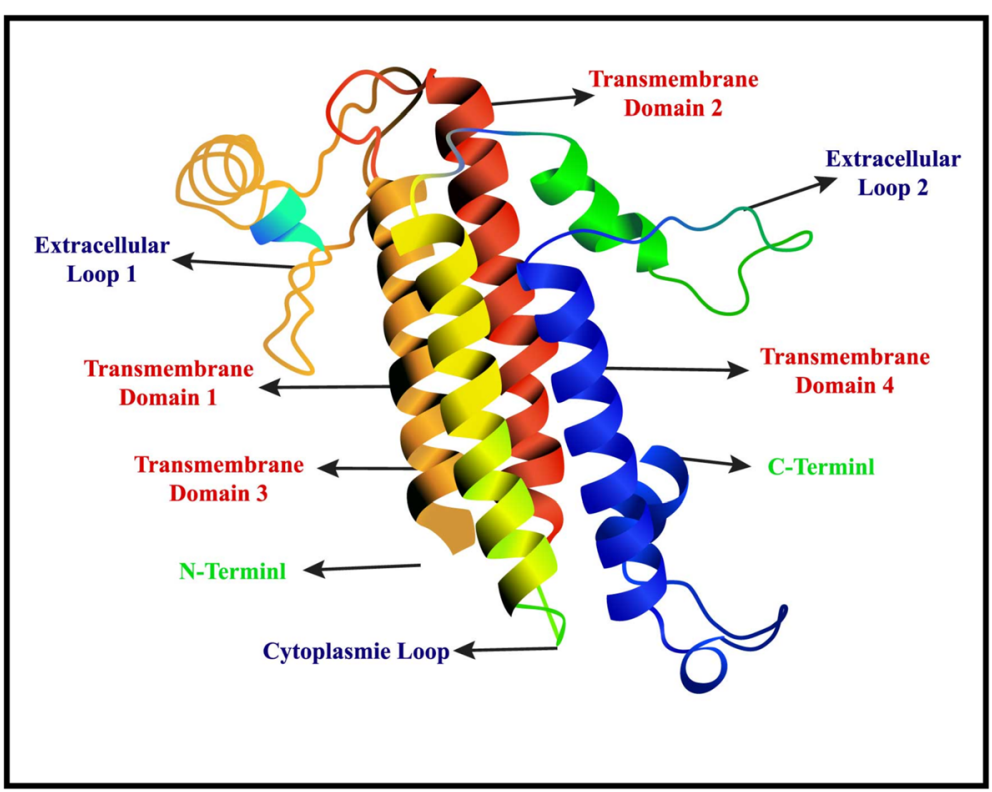

Fig. 1 Model structure of claudin protein

Ugur Sahin et al. identified isoform 2 of the tight junction molecule claudin-18 (CLDN18.2) as a highly selective cell lineage marker [23].They observed its expression in normal tissues is strictly confined to differentiated epithelial cells of the gastric mucosa, but it was absent from the gastric stem cell zone. Claudin 18.2 was retained on malignant transformation and was expressed in a significant proportion of primary gastric cancers and its metastases. Frequently ectopic activation of claudin 18.2 was also found in pancreatic, esophageal, ovarian, and lung tumors. The study suggested that CLDN18.2 has highly restricted expression pattern in normal tissues, with frequent ectopic activation in a diversity of human cancers.

Claudin 18.2 is involved in tumor development and progression and located in the outer cell membrane. It has exposed extracellular loops and is available for monoclonal antibody binding. These biological characteristics suggested that it is an ideal molecule for targeted therapy and led to the further development of monoclonal antibodies against claudin 18.2, such as claudiximab (IMAB362).

\section{Claudiximab (IMAB362)}

Claudiximab is a novel chimeric IgG1 antibody highly specific for claudin 18.2. Claudiximab is derived from a murine monoclonal antibody and has been chimerized to display the human IgG1 constant region for clinical use. Claudiximab is produced in Chinese Hamster Ovary $(\mathrm{CHO})$ cells by standard recombinant expression technology. It binds to claudin 18.2 on the tumor cell surface to stimulate cellular and soluble immune effectors that activate antibody-dependent cytotoxicity (ADCC) and complement dependent cytotoxicity (CDC). It can also induce apoptosis and inhibit cell proliferation. When combined with chemotherapy, claudiximab enhances Tcell infiltration and induce pro-inflammatory cytokines (Fig. 2).

The safety of the claudiximab was extensively assessed in preclinical animal models. In preclinical studies, claudiximab proved to inhibit tumor growth and eradicate cancer cells by mechanisms such as complement-dependent cytotoxicity $(\mathrm{CDC})$ and antibody-dependent cellular cytotoxicity (ADCC) [24].

\section{Claudiximab for advanced gastric cancer treatment in clinical trials}

Currently, claudiximab is studied in numerous clinical trials for the treatment of patients with advanced gastroesophageal cancer. Data reported in the clinical trials of claudiximab are summarized in Table 1.

The first in-human phase I single-dose escalation study enrolled 15 patients with treatment-refractory metastatic gastroesophageal adenocarcinomas with CLDN18.2positive tumors as determined by CLAUDETECT ${ }^{\mathrm{Tm}} 18.2$ Kit by immunohistochemistry test [24]. Patients were enrolled into five dose groups from 33 to $1000 \mathrm{mg} / \mathrm{m}^{2}$ with three patients in each group. Patients were followed up for 4 weeks. If disease control was achieved, claudiximab was allowed to be continued until progression. Phase I trial reported all dose levels of claudiximab were generally well tolerated with nausea, and vomiting were the most common adverse events (AEs). No dose-limiting toxicity was observed at single doses up to $1000 \mathrm{mg} / \mathrm{m}^{2}$. Based on pharmacokinetic considerations and preclinical dose/ 


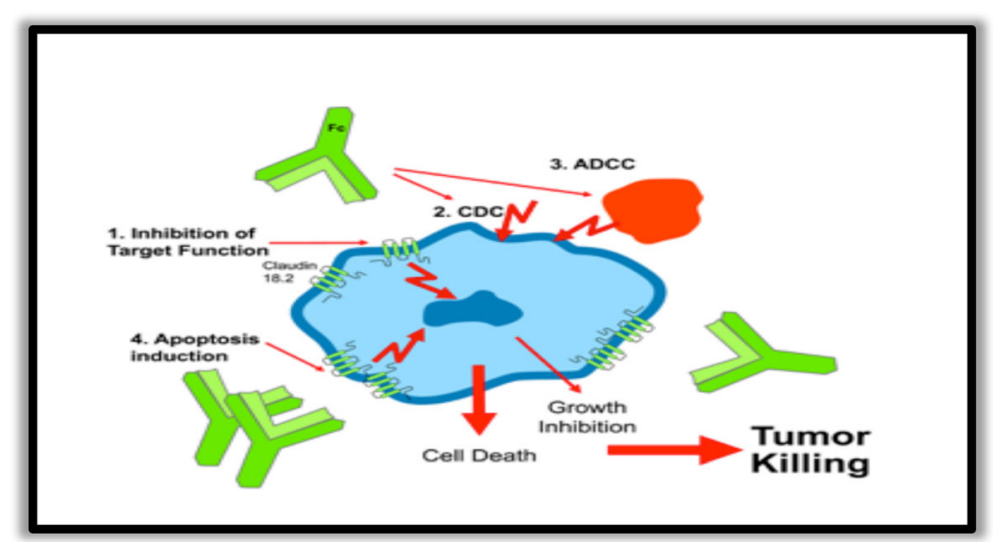

Fig. 2 Mechanism of action of claudiximab (IMAB362)

response data, a $600 \mathrm{mg} / \mathrm{m}^{2}$ dose was recommended for the phase II multidose trial (NCT00909025).

In another phase I trial "PILOT" evaluated the safety and efficacy of claudiximab in combination with zoledronic acid (ZA) and interleukin-2(IL2) in patients with CLDN18.2+ gastroesophageal adenocarcinomas [25]. Twenty-eight patients who had progressed on more than one chemotherapy regimen were enrolled and received claudiximab $(800 \mathrm{mg} / \mathrm{m} 2$ in cycle [cy] 1 , followed by $600 \mathrm{mg} / \mathrm{m} 2$ in subsequent cy, IV q3w) plus ZA (4 mg, IV, day 1 of cy 1 and 3 ) in arm 1; claudiximab, ZA plus low IL-2 $\left(1 \times 10^{6} \mathrm{IU}\right.$, s.c., days $1-3$ of cy 1 and 3$)$ in arm 2; claudiximab, ZA plus intermediate IL-2 $\left(3 \times 10^{6} \mathrm{IU}\right.$ s.c., days $1-3$ of cy 1 and 3 ) in arm 3; and IMAB362 alone in arm 4. Of the 20 evaluable patients, 11 patients achieved disease control, 1 patient had unconfirmed partial response; the rest 10 patients had stable disease. The median progression-free survival was 12.7 weeks, and median overall survival was 40 weeks. Claudiximab alone and in combination was well tolerated and common treatment-related adverse events (AEs) included nausea and vomiting mostly grades $1-3$. The study concluded that claudiximab has anti-tumor activity as monotherapy and can also be safely combined with ZA/
IL2 in the treatment of advanced gastroesophageal cancers (NCT01671774).

This was followed by a phase IIa (MONO) study aimed at establishing the efficacy and safety of multiple doses of claudiximab as monotherapy in patients with metastatic, refractory, or recurrent adenocarcinoma of the stomach or the lower esophagus [26]. The study enrolled 54 patients; with four patients receiving biweekly $300 \mathrm{mg} / \mathrm{m}^{2}$ and 50 receiving $600 \mathrm{mg} / \mathrm{m}^{2}$ biweekly as 2 hour infusion. Patients with disease control $(N=9)$ were allowed to continue claudiximab therapy until progression. Forty patients all in the $600 \mathrm{mg} / \mathrm{m}^{2}$ were evaluable for analysis. The response rate was $10 \%$, and the disease control rate was $30 \%$ (best observed response: PR, $n=4$ and SD, $n=8)$. Median PFS was 102 days $(95 \%$ CI, 70 146 days). All observed AEs were grades $1-3$. The most frequent grade 3 AEs were vomiting in 31 pts. No grade 4 AEs occurred. Pharmacokinetics from the study supported 3-weekly IV dosing (NCT01197885).

Subsequently, a phase IIb (FAST) study evaluated claudiximab as first line in patients with advanced/recurrent gastric and GEJ cancer. Patients with CLDN18.2 expression of $\geq 2+$ in $\geq 40 \%$ tumor cells (as validated by CLAUDETECT $^{\mathrm{sm}}$ 18.2 Kit), Eastern Cooperative Oncology Group

Table 1 Various clinical trials involving claudiximab (IMAB362)

\begin{tabular}{|c|c|c|c|c|c|c|c|c|}
\hline $\begin{array}{l}\text { Study-completion } \\
\text { date }\end{array}$ & NCT number & Phase & $\begin{array}{l}\text { Number of } \\
\text { patients }\end{array}$ & Design & Response rate & OS & $\begin{array}{l}\text { Median } \\
\text { FPS }\end{array}$ & $\begin{array}{l}\text { Adverse } \\
\text { events }\end{array}$ \\
\hline- & NCT00909025 & I & 15 & $\begin{array}{l}\text { Single-dose escalation } \\
\text { safety study }\end{array}$ & - & - & - & - \\
\hline PILOT-2014 & NCT01671774 & I & 20 & $\begin{array}{l}\text { Multiple dose, } \\
\text { immunomodulation IMAB362 } \\
\text { + zoledronic acid + IL-2 }\end{array}$ & $\begin{array}{l}11 \text { patients had } \\
\text { disease control }\end{array}$ & 40 weeks & 12.7 weeks & $\begin{array}{l}\text { Nausea and } \\
\text { vomiting }\end{array}$ \\
\hline MONO-2013 & NCT01197885 & $\| \mathrm{A}$ & 54 & $\begin{array}{l}\text { Single arm, repeated dose, } \\
\text { monotherapy study }\end{array}$ & $\begin{array}{l}\text { Response rate-10\% } \\
\text { Disease control } \\
\text { rate-30\% }\end{array}$ & - & 14.5 weeks & Vomiting \\
\hline FAST-2015 & NCT01630083 & $\| B$ & $161+85$ & $\begin{array}{l}\text { Randomized EOX vs claudiximab } \\
+ \text { EOX, extended with high-dose } \\
\text { claudiximab + EOX }\end{array}$ & $\begin{array}{l}\text { Objective response } \\
\text { rate- } 25 \text { vs 39\% }\end{array}$ & $\begin{array}{l}8.4 \text { vs } 13.4 \\
\text { months }\end{array}$ & $\begin{array}{l}4.8 \text { vs } 7.9 \\
\text { months }\end{array}$ & $\begin{array}{l}\text { Vomiting, } \\
\text { neutrope-nia, } \\
\text { and anemia }\end{array}$ \\
\hline
\end{tabular}


performance status of $0-1$ and who were not eligible for trastuzumab were included in the study [27, 28]. Seven hundred thirty-nine patients were consented and 352 (48\%) were tested CLDN18.2+ per protocol criteria. Of those, 161 patients (gastric, 80\%; GEJ, 16\%; esophageal, 4\%) were randomized in 1:1 to first-line EOX (epirubicin $50 \mathrm{mg} /$ $\mathrm{m} 2$, oxaliplatin $130 \mathrm{mg} / \mathrm{m} 2 \mathrm{~d} 1$, and capecitabine $625 \mathrm{mg} / \mathrm{m} 2$ bid, d1-21, every 21 days) with or without claudiximab (loading dose $800 \mathrm{mg} / \mathrm{m} 2$, then $600 \mathrm{mg} /$ $\mathrm{m} 2 \mathrm{~d} 1$, every 21 days). The study had an exploratory arm three $(N=85)$ to investigate a high-dose claudiximab $(1000 \mathrm{mg} / \mathrm{m} 2)$ plus EOX. The study met its primary end point of progression-free survival (PFS). Claudiximab plus EOX significantly improved PFS (median 7.9 vs 4.8 months; HR $0.47 ; p=0.0001$ ) and OS (median 13.3 vs 8.4 months; HR $0.51, p<0.001$ ) compared to EOX alone. Subgroup analysis of patients with very high CLDN18.2 expression ( $\geq 2+$ intensity in $\geq 70 \%$ tumor cells), outcomes were more pronounced (PFS, 7.2 vs 5.6 months; HR 0.36; $p=0.0005$; OS, 9.0 vs 16.7 months; HR $0.45, p<$ $0.0005)$. Patients who received claudiximab also showed a higher objective response rate (ORR) at 39\% compared with $25 \%$ in the EOX arm. In the claudiximab cohort, 8 patients $(10.4 \%)$ achieved a complete response (CR), 22 patients $(28.6 \%)$ had a partial response (PR), and 34 patients (44.2\%) had stable disease (SD). With chemotherapy, 3 patients (3.6\%) achieved a CR, 18 (21.4\%) had a PR, and $43(51.2 \%)$ had SD. Disease progression was experienced by 5.2 and $11.9 \%$ of patients receiving claudiximab vs chemotherapy, respectively. The treatment was well tolerated with mostly grade 1/2-related adverse events which included vomiting, neutropenia, and anemia. Grade $3 / 4$ events were not significantly increased in patients receiving claudiximab. Overall, $55.8 \%$ of patients in the investigational cohort showed grades $1 / 2$ vomiting, and $10.4 \%$ had grades $3 / 4$ events compared with the chemotherapy arm, wherein $34.5 \%$ of patients had grades $1 / 2$ vomiting and $3.6 \%$ had grades $3 / 4$ events. The incidence and severity of vomiting seems dose-dependent. Vomiting experienced by patients was proportional to the dose of claudiximab administered. Vomiting was reported in 60 to $69 \%$ of patients receiving medium and experimental doses of claudiximab compared with $<40 \%$ of patients on chemotherapy alone. The investigators concluded that claudiximab in combination with first line chemotherapy provided clinically relevant benefit in PFS and OS in patients with CLDN18.2 positive gastric and GEJ adenocarcinoma (NCT01630083).

\section{Testing for claudin 18.2 positivity: CLAUDETECT $^{\mathrm{Tm}}$ 18.2}

All the above studies showed benefits in the patients with tumor positive for CLDN18.2 expression of $\geq 2+$ in $\geq 40 \%$ tumor cells. Of note, only $48 \%$ of the patients in the FAST study were considered positive for CLDN18.2 [28]. The biomarker's presence in tumor specimens was determined by CLAUDETECT ${ }^{\mathrm{Tu}} 18.2$, a pathology test developed by Ganymed (now acquired by Astellas), the drug maker and sponsor of the trial. CLAUDETECT ${ }^{\mathrm{mat}} 18.2$ is a semi-quantitative immunohistochemical assay that selectively and exclusively determines CLDN18.2 protein expression in formalin-fixed, paraffin-embedded tumor tissues from patients with adenocarcinoma of the stomach, gastroesophageal junction, and pancreas as well as bile duct and lung. CLAUDETECT ${ }^{\mathrm{mm}} 18.2$ is the first and only CE marked in vitro diagnostic (IVD) test which allows to assess the expression levels of claudin 18.2 (CLDN18.2) in gastroesophageal, pancreatic, lung, and other solid tumors.

\section{Future outlook}

The major limitation will be the commercial easy availability of the testing for CLDN18.2. The need for further studies revolves around finding the ideal cut-off point for the CLDN18.2 levels, which suggest the need for studies comparing outcomes between low CLDN18.2 levels vs higher levels are warranted. Another notable point in the FAST study is that the outcomes in the EOX only arm were not similar to the landmark trial REAL 2 study [OS of 11.2 (REAL 2) vs 8.7 (FAST)] [29], which could be due to patient selection. In addition, it may be interesting to know the outcomes of claudiximab-based chemotherapy compared to other regimens including Her2 directed therapy.

\section{Conclusions}

Targeted therapy is clearly the future of gastric cancer treatments. Newer concepts must implement multi-target strategies in order to induce durable remissions. Claudiximab is a novel recombinant chimeric monoclonal antibody, which has demonstrated a significant efficacy and safety profiles when combined with other standard chemotherapy drugs and as a monotherapy in gastro-esophageal cancer in early clinical trials. It is a very promising targeted therapy for these difficult-to-treat malignancies. A phase III trial is scheduled to be started in 2017, which hopefully will provide more definitive answers and bring this agent closer to approval by the drug regulatory agencies.

\footnotetext{
Abbreviations

ADCC: Antibody-dependent cytotoxicity; AEs: Adverse events;

CDC: Complement dependent cytotoxicity; CE: Conformité Européene; CLDN: Claudin; CR: Complete response; EOX: Epirubicin, oxaliplatin, Xeloda (capecitabine); IL2: Interleukin-2; IMAB: Ideal monoclonal antibody; ORR: Objective response rate; PFS: Progression-free survival; PR: Partial response; RR: Response rate; SD: Stable disease; ZA: Zoledronic acid
}

\section{Acknowledgements}

Not applicable.

Funding

Not applicable. 


\section{Availability of data and materials}

The datasets used and/or analyzed during the current study are available from the corresponding author on reasonable request.

\section{Authors' contributions}

PS analyzed the available data, drafted the manuscript, and critically analyzed the manuscript. ST drafted the manuscript. YH analyzed the available data and critically analyzed the manuscript. All authors have read and approved the final manuscript.

\section{Competing interests}

The authors declare that they have no competing interests.

\section{Consent for publication}

Not applicable.

\section{Ethics approval and consent to participate} Not applicable.

\section{Publisher's Note}

Springer Nature remains neutral with regard to jurisdictional claims in published maps and institutional affiliations.

\section{Author details \\ ${ }^{1}$ Hematology/Oncology Fellowship Program, Maimonides Medical Center, Brooklyn, NY, USA. ${ }^{2}$ Department of Internal Medicine, Maimonides Medical Center, Brooklyn NY, USA. ${ }^{3}$ Division of Hematology/Oncology, Maimonides Medical Center, Brooklyn, NY, USA.}

\section{Received: 25 March 2017 Accepted: 29 April 2017}

\section{Published online: 12 May 2017}

\section{References}

1. Torre LA, Bray F, Siegel RL, Ferlay J, Lortet-Tieulent J, Jemal A. Global cancer statistics, 2012. CA Cancer J Clin. 2015;65(2):87-108.

2. Buzzoni R, Bajetta E, Di Bartolomeo M, Miceli R, Beretta E, Ferrario E, Mariani L. Pathological features as predictors of recurrence after radical resection of gastric cancer. Br J Surg. 2006;93(2):205-9.

3. Macdonald JS, Smalley SR, Benedetti J, Hundahl SA, Estes NC, Stemmermann GN, Haller DG, Ajani JA, Gunderson LL, Jessup JM, et al. Chemoradiotherapy after surgery compared with surgery alone for adenocarcinoma of the stomach or gastroesophageal junction. N Engl J Med. 2001;345(10):725-30.

4. Wagner AD, Unverzagt S, Grothe W, Kleber G, Grothey A, Haerting J, Fleig WE. Chemotherapy for advanced gastric cancer. Cochrane Database Syst Rev. 2010;3:CD004064.

5. Bang YJ, Van Cutsem E, Feyereislova A, Chung HC, Shen L, Sawaki A, Lordick F, Ohtsu A, Omuro Y, Satoh T, et al. Trastuzumab in combination with chemotherapy versus chemotherapy alone for treatment of HER2-positive advanced gastric or gastro-oesophageal junction cancer (ToGA): a phase 3, open-label, randomised controlled trial. Lancet. 2010;376(9742):687-97.

6. Wilke H, Muro K, Van Cutsem E, Oh SC, Bodoky G, Shimada Y, Hironaka S, Sugimoto N, Lipatov O, Kim TY, et al. Ramucirumab plus paclitaxel versus placebo plus paclitaxel in patients with previously treated advanced gastric or gastro-oesophageal junction adenocarcinoma (RAINBOW): a doubleblind, randomised phase 3 trial. Lancet Oncol. 2014;15(11):1224-35.

7. Ansell SM, Lesokhin AM, Borrello I, Halwani A, Scott EC, Gutierrez M, Schuster SJ, Millenson MM, Cattry D, Freeman GJ, et al. PD-1 blockade with nivolumab in relapsed or refractory Hodgkin's lymphoma. N Engl J Med. 2015;372(4):311-9.

8. Brahmer J, Reckamp KL, Baas P, Crino L, Eberhardt WE, Poddubskaya E, Antonia S, Pluzanski A, Vokes EE, Holgado E, et al. Nivolumab versus docetaxel in advanced squamous-cell non-small-cell lung cancer. N Engl J Med. 2015;373(2):123-35

9. Garon EB, Rizvi NA, Hui R, Leighl N, Balmanoukian AS, Eder JP, Patnaik A, Aggarwal C, Gubens M, Horn L, et al. Pembrolizumab for the treatment of non-small-cell lung cancer. N Engl J Med. 2015;372(21):2018-28.

10. Larkin J, Chiarion-Sileni V, Gonzalez R, Grob JJ, Cowey CL, Lao CD, Schadendorf D, Dummer R, Smylie M, Rutkowski P, et al. Combined nivolumab and ipilimumab or monotherapy in untreated melanoma. N Engl J Med. 2015;373(1):23-34.
11. Postow MA, Chesney J, Pavlick AC, Robert C, Grossmann K, McDermott D, Linette GP, Meyer N, Giguere JK, Agarwala SS, et al. Nivolumab and ipilimumab versus ipilimumab in untreated melanoma. N Engl J Med. 2015; 372(21):2006-17.

12. Furgan M, Mukhi N, Lee B, Liu D. Dysregulation of JAK-STAT pathway in hematological malignancies and JAK inhibitors for clinical application. Biomark Res. 2013;1 (1):5.

13. Yu J, Zhang $Y$, Leung $L H$, Liu L, Yang F, Yao X. Efficacy and safety of angiogenesis inhibitors in advanced gastric cancer: a systematic review and meta-analysis. J Hematol Oncol. 2016;9(1):111.

14. Wang X, Wang C, Zhang X, Hua R, Gan L, Huang M, Zhao L, Ni S, Guo W. Bmi-1 regulates stem cell-like properties of gastric cancer cells via modulating miRNAs. J Hematol Oncol. 2016;9(1):90.

15. Liu J, Pan C, Guo L, Wu M, Guo J, Peng S, Wu Q, Zuo Q. A new mechanism of trastuzumab resistance in gastric cancer: MACC1 promotes the Warburg effect via activation of the PI3K/AKT signaling pathway. J Hematol Oncol. 2016;9(1):76

16. Goel G, Sun W. Advances in the management of gastrointestinal cancers - an upcoming role of immune checkpoint blockade. J Hematol Oncol. 2015;8:86.

17. Furuse M, Fujita K, Hiiragi T, Fujimoto K, Tsukita S. Claudin-1 and -2: novel integral membrane proteins localizing at tight junctions with no sequence similarity to occludin. J Cell Biol. 1998;141(7):1539-50.

18. Swisshelm K, Macek R, Kubbies M. Role of claudins in tumorigenesis. Adv Drug Deliv Rev. 2005;57(6):919-28.

19. Morin PJ. Claudin proteins in human cancer: promising new targets for diagnosis and therapy. Cancer Res. 2005;65(21):9603-6.

20. Resnick MB, Konkin T, Routhier J, Sabo E, Pricolo VE. Claudin-1 is a strong prognostic indicator in stage II colonic cancer: a tissue microarray study. Mod Pathol. 2005;18(4):511-8.

21. Sanada Y, Oue N, Mitani Y, Yoshida K, Nakayama H, Yasui W. Downregulation of the claudin-18 gene, identified through serial analysis of gene expression data analysis, in gastric cancer with an intestinal phenotype. J Pathol. 2006;208(5):633-42.

22. Cheung ST, Leung KL, Ip YC, Chen X, Fong DY, Ng IO, Fan ST, So S. Claudin10 expression level is associated with recurrence of primary hepatocellular carcinoma. Clin Cancer Res. 2005;11(2 Pt 1):551-6.

23. Sahin U, Koslowski M, Dhaene K, Usener D, Brandenburg G, Seitz G, Huber C, Tureci O. Claudin-18 splice variant 2 is a pan-cancer target suitable for therapeutic antibody development. Clin Cancer Res. 2008;14(23):7624-34.

24. Schuler MH, Zvirbule Z, Lordick F, Krilova A, Helbig U, Schulze-Bergkamen H, Thuss-Patience P-C, Wichert G-V, Schulmann K, Trarbach T, Bauer S, Mueller C, AlBatran S-E, Huber C, Sahin U, Tureci O. Safety, tolerability, and efficacy of the firstin-class antibody IMAB362 targeting claudin 18.2 in patients with metastatic gastroesophageal adenocarcinomas. ASCO Meet Abstr. 2013;31:4080.

25. Sahin U, Al-Batran S-E, Hozaeel W, Zvirbule Z, Freiberg-Richter J, Lordick F, Just M, Bitzer M, Thuss-Patience P-C, Krilova A, Brass V, Mueller C, Daalman E, Tureci O. IMAB362 plus zoledronic acid (ZA) and interleukin-2 (LL-2) in patients (pts) with advanced gastroesophageal cancer (GEC): clinical activity and safety data from the PILOT phase I trial. ASCO Meet Abstr. 2015;33:e15079.

26. Trarbach T, Schuler M, Zvirbule Z, Lordick F, Krilova A, Helbig U, SchulzeBergkamen H, Thuss-Patience P-C, Wichert G, Schmiegel W, Bauer S, Müller C, Al-Batran S, Huber C, Maurus D, Kühnle M, Sahin U, Türeci O. 636P. Efficacy and safety of multiple doses of IMAB362 in patients with advanced gastroesophageal cancer: results of a phase II study. Ann Oncol. 2014;25(4 Supplement):iv218.

27. Al-Batran S-E, Schuler M, Zvirbule Z, Manikhas G, Lordick F, Tureci O, Huber C, Sahin U. LBA-06. IMAB362: a novel immunotherapeutic antibody targeting the tight-junction protein component CLAUDIN18.2 in gastric cancer. Ann Oncol. 2016;27(2 Supplement):ii141-2.

28. Al-Batran S, Schuler M, Zvirbule Z, Manikhas G, Lordick F, Rusyn A, Vynnyk Y, Vynnychenko I, Fadeeva N, Nechaeva M, Dudov A, Gotovkin E, Pecheniy A, Bazin I, Bondarenko I, Melichar B, Mueller C, Huber C, Tureci O, Sahin U. FAST: an international, multicenter, randomized, phase II trial of epirubicin, oxaliplatin, and capecitabine (EOX) with or without IMAB362, a first-in-class anti-CLDN18.2 antibody, as first-line therapy in patients with advanced CLDN18.2+ gastric and gastroesophageal junction (GEJ) adenocarcinoma. ASCO Meet Abstr. 2016;34:LBA4001.

29. Cunningham D, Starling N, Rao S, Iveson T, Nicolson M, Coxon F, Middleton G, Daniel F, Oates J, Norman AR, et al. Capecitabine and oxaliplatin for advanced esophagogastric cancer. N Engl J Med. 2008;358(1):36-46. 